\title{
JOINT HYDROLOGICAL-GEOPHYSICAL INVERSION FOR SOIL STRUCTURE IDENTIFICATION
}

\author{
Stefan Finsterle and Michael B. Kowalsky \\ Lawrence Berkeley National Laboratory \\ Earth Sciences Division \\ 1 Cyclotron Road, Mail Stop 90-1116 \\ Berkeley, CA 94720, U.S.A. \\ e-mail: SAFinsterle@lbl.gov
}

\begin{abstract}
Reliable prediction of subsurface flow and contaminant transport depends on the accuracy with which the values and spatial distribution of process-relevant model parameters can be identified. Successful characterization methods for complex soil systems are based on (1) an adequate parameterization of the subsurface, capable of capturing both random and structured aspects of the heterogeneous system, and (2) site-specific data that are sufficiently sensitive to the processes of interest. We present a stochastic approach where the high-resolution imaging capability of geophysical methods is combined with the process-specific information obtained from the inversion of hydrological data. Geostatistical concepts are employed as a flexible means to describe and characterize subsurface structures. The key features of the proposed approach are (1) the joint inversion of geophysical and hydrological raw data, avoiding the intermediate step of creating a (non-unique and potentially biased) tomogram of geophysical properties, (2) the concurrent estimation of hydrological and petrophysical parameters in addition to (3) the determination of geostatistical parameters from the joint inversion of hydrological and geophysical data; this approach is fundamentally different from inference of geostatistical parameters from an analysis of spatially distributed property data. The approach has been implemented into the iTOUGH2 inversion code and is demonstrated for the joint use of synthetic timelapse ground-penetrating radar (GPR) travel times and hydrological data collected during a simulated ponded infiltration experiment at a highly heterogeneous site.
\end{abstract}

\section{INTRODUCTION}

It is recognized that subsurface flow and contaminant transport processes are critically affected by the soil structure and heterogeneity of the subsurface as well as the related distribution of soil moisture. While geophysical methods-such as ground penetrating radar (GPR) - may provide high-resolution images of the subsurface, the relation between these images and parameters affecting flow and transport remains ambiguous. On the other hand, although hydrological data contain information about properties relevant to flow and transport, their spatial coverage and resolution are usually insufficient to capture the features governing the system behavior. A joint inversion approach (in which geophysical and hydrological data are inverted concurrently to obtain high-resolution models of hydrologic parameters and soil moisture distribution) has the potential to combine the strengths of both characterization methods.

The difficulty of predicting unsaturated flow and transport in porous or fractured media stems largely from the development of preferential flow patterns, which are mostly induced by heterogeneities of hydrologic properties on multiple scales [Pruess et al., 1999a]. Ignoring such heterogeneities in a simulation model leads to a volume-averaged behavior that does not adequately capture the highly localized water flow occurring along preferential pathways. Methods have been developed to describe and simulate heterogeneity with various degrees of randomness, spatial correlation, and inclusion of deterministic structures [Deutsch and Journel, 1992; McLaughlin and Townley, 1996; Zimmerman et al., 1998; Yeh and Šimunek, 2002; Finsterle, 2005]. In these methods, the random component of the heterogeneity is often described by a stochastic model with parameterized distributional assumptions about the spatial variability of the property. These parameters are then estimated, and geostatistical simulation and ensemble averaging are employed to identify the subsurface structure and its uncertainty. It should be noted that this averaging process may again lead to an overly smooth model, which potentially masks the preferential flow patterns that lead to early contaminant breakthrough. To perform accurate site-specific simulations, it is therefore crucial to identify realizations of the property field that are as close to the actual distribution as possible. This requires an approach that determines hydrologic properties over the relevant domain directly rather than through interpolation or geostatistical simulation. As mentioned above, geophysical imaging has the 
potential to provide the needed high-resolution information.

Geophysical data have been routinely used for the development of hydrological models. Most commonly, geophysical data are inverted to create images that are used to identify the soil stratigraphy, which is then assigned to zones with constant hydrological properties. These properties may be determined by the inversion of hydrological data. In this sequential approach, any artifact in the geophysical inversion or misinterpretation of the geophysical image leads to an error in the conceptual model, which in turn biases the estimates of the hydrological parameters and potentially corrupts the model predictions.

Certain geophysical data, such as GPR travel times, are sensitive to changes in the water content, and can thus be used to measure soil water content [Huisman et al., 2003], provided that an accurate petrophysical model is given that relates the composite dielectric constant to the volumetric water content. This approach allows for state estimation, and if the timelapse tomography is employed, the changes in the inferred water content distribution can be the basis for hydrological interpretation [Binley et al., 2002]. The water content maps can also be used-as derived data - in a formal calibration of a hydrological model. This approach relies again on a tomographic inversion and also an accurate petrophysical relationship, the parameters of which may be determined by the inversion of data from laboratory experiments. To avoid the related pitfalls (inversion artifacts, errors in the petrophysical model, and scaling issues), geophysical and hydrological raw data can be analyzed simultaneously in a joint inversion, in which a geophysical and hydrological forward model are linked and used as part of an algorithm that minimizes the differences between calculated and measured data [Kowalsky et al., 2004; Lambot et al., 2004; Rucker and Ferré, 2004; Kowalsky et al., 2005]. This approach combines parameter and state estimation. Moreover, the use of complementary hydrological and geophysical data may reduce the inherent ambiguity and nonuniqueness of the inverse problem.

In this paper, we expand our previously published joint inversion approach [Kowalsky et al., 2004; 2005] to include concurrent estimation of geostatistical parameters in an attempt to identify the soil structure along with the physical parameters. In this approach, geostatistical parameters are determined simultaneously with hydrological and petrophysical parameters by jointly inverting geophysical and hydrological data. It should be noted that this is fundamentally different from inference of geostatis- tical parameters from a direct analysis of spatially distributed property data. While such property data can be included in the inversion as prior information, their availability is not a condition of the method. Instead, the geostatistical parameters are inferred from the impact the property field has on water flow through the vadose zone, which in turn is reflected by the measured hydrological and geophysical data. A key presumption of the approach is that a suitable parametric model can be found that is flexible enough to describe both the random and deterministic aspects of the soil structure. In this demonstration, we rely on standard geostatistical simulation techniques to create random spatial structures that are conditioned on property values to be estimated at selected pilot points [RamaRao et al., 1995; Gomez-Hernandez et al., 1997].

We first discuss the hydrological and geophysical forward models, followed by the parameterization of the soil structure to make it amenable to joint inversion. Finally, we demonstrate the approach using synthetic time-lapse ground-penetrating radar (GPR) travel times and hydrological data collected during a ponded infiltration experiment at a highly heterogeneous site.

\section{HYDROLOGICAL FORWARD MODEL}

We are concerned with water flow through the vadose zone, which can be described using Richards' equation [Richards, 1931] as implemented in the integral finite-difference simulator TOUGH2 [Pruess et al., 1999b]:

$$
\frac{\partial}{\partial t} \phi S \rho=\nabla\left[k \frac{k_{r}}{\mu} \rho \nabla(P+\rho g z)\right]
$$

Here, $t$ is time, $\phi$ is porosity, $S$ is liquid saturation, $\rho$ is liquid density, $k$ is absolute permeability, $k_{r}$ is relative permeability, $\mu$ is viscosity, $g$ is gravitational acceleration, $z$ is the vertical coordinate (positive upward), and $P=P_{\text {ref }}+P_{c}$ is liquid-phase pressure, where $P_{r e f}$ is a reference gas pressure and $P_{c}$ is capillary pressure. Relative permeability and capillary pressure are functions of liquid saturation as given by van Genuchten [1980]:

$$
\begin{gathered}
k_{r}=S_{e}^{1 / 2}\left[1-\left(1-S_{e}^{1 / m}\right)^{m}\right]^{2} \\
P_{c}=-\frac{1}{\alpha}\left[S_{e}^{-1 / m}-1\right]^{1-m}
\end{gathered}
$$

where the effective saturation $S_{e}$ is defined as:

$$
S_{e}=\frac{S-S_{l r}}{1-S_{l r}}
$$


In the van Genuchten-Mualem model, $S_{l r}$ is the residual liquid saturation, and $\alpha$ and $m$ are fitting parameters. The capillary-strength parameter $1 / \alpha$ appearing in (3) is assumed to be correlated to the heterogeneous permeability field according to Leverett's scaling rule [Leverett, 1941]:

$$
\frac{1}{\alpha_{n}}=\frac{1}{\alpha_{r e f}}\left(\frac{k_{r e f}}{k_{n}}\right)^{1 / 2}
$$

Here, $k_{n}$ and $1 / \alpha_{n}$ are, respectively, the permeability and the capillary-strength parameter of gridblock $n$ in the numerical model, $k_{r e f}$ is the reference permeability, and $1 / \alpha_{\text {ref }}$ is the reference capillary-strength parameter, both being determined in the joint inversion process. Residual liquid saturation $S_{l r}$ was set to 0.08 , and the parameter $m$ was chosen to be 0.63 .

\section{GEOPHYSICAL FORWARD MODEL}

For computational efficiency, we simulate GPR travel times using the straight-ray method, which is based on a high-frequency approximation that calculates the arrival time of the first amplitude departure of the transmitted wave, ignoring the remainder of the waveform. As demonstrated in Kowalsky et al. [2005], the errors introduced by assuming the GPR energy travels along a straight path does not, in many cases, significantly impact the estimated parameters and predicted system behavior for the system considered in this study. In the straight-ray method, the travel time $T$ for an electromagnetic (EM) wave propagating between the transmitting and receiving antennas can be calculated by connecting the antennas with a straight line and summing the travel times of the EM wave as it travels through the discretized model domain:

$$
T=\sum_{i=1}^{N} \frac{L_{i}}{C}\left[\left(1-\phi_{i}\right) \kappa_{s}^{n}+S_{i} \phi_{i} \kappa_{w}^{n}+\left(1-S_{i}\right) \phi_{i} \kappa_{a}^{n}\right]^{1 / 2 n}
$$

Here, $L_{i}$ is the length of the linear travel segment in block $i, N$ is the number of blocks through which the ray passes, and $c$ is the EM wave velocity in free space. The term in brackets results from a volumetric mixing formula [Roth et al., 1990] used as the petrophysical function that calculates the effective dielectric constant from the local water saturation $S_{i}$, porosity $\phi_{i}$, and the dielectric constants of the individual phases (with $\kappa_{s}$ being the dielectric constant for the solid, and $\kappa_{w}$ and $\kappa_{a}$ the known dielectric constants for water and air, respectively). The exponent $n$ is related to the geometric arrangement of materials relative to the applied electric field [Ansoult et al., 1984].

\section{PARAMETERIZATION OF SOIL STRUCTURE}

Geological structures or stratal soil architectures are results of complex geologic processes (deposition, erosion, deformation, intrusion, fracturing, etc.). Depending on the scale of observation, these processes and the resulting morphology can be considered to be deterministic, random, or a combination of the two, which is reflected in the emergence of hierarchical structures with randomly distributed attributes that exhibit both spatial continuity and discrete interfaces. Characterization of these subsurface structures is an attempt to capture the underlying complexity with a few categorical terms or parameters that are part of a descriptive model. To handle the randomness inherent in the subsurface, almost all of these models are stochastic in nature. Among the most widely applied techniques are semivariogram-based geostatistical models [Deutsch and Journel, 1992] and concepts relying on the fractal distribution of soil properties [Neuman, 1990; Molz et al., 1997]. Sediments can also be described with a hierarchical transition probability model [Carle and Fogg, 1996], with relative proportions of lithofacies and mean and variance of their lengths being the key parameters.

We currently rely on geostatistical simulation as a flexible means to generate subsurface structures that exhibit (1) small-scale randomness (on the grid-block scale) and (2) some medium-scale spatial continuity (on a scale larger than a grid block but smaller than the size of the domain of interest). These two scales of heterogeneity capture two significant controls on subsurface flow and transport. Moreover, the targeted resolution is comparable to the resolution of geophysical methods and is on a scale on which effective flow parameters can be reasonably defined. Note that using geostatistical simulation (instead of interpolation techniques such as kriging) enables small-scale heterogeneities to be included in the model (even though only in a stochastic sense). This feature counters the tendency of overdetermined or regularized inverse solutions to smooth out the property field.

Modeling of site-specific spatial variability by geostatistical simulation essentially consists of estimating a property field using sampled values and some prior knowledge about the spatial correlation of the attribute. The latter is usually expressed through a semivariogram. In our approach, neither the values at the sampling points nor the semivariogram parameters are assumed to be known; instead, they are estimated as part of the joint inversion of geophysical and hydrological data. Estimating property values at discrete locations for use as conditioning points in the subsequent geostatistical simulation is part of the 
pilot point method described by RamaRao et al. [1995] and Gomez-Hernandez et al. [1997]. We expand this concept by concurrently estimating semivariogram parameters, such as the correlation length, anisotropy ratio, and orientation; we assume the functional form of the semivariogram as well as the sill value to be given. This framework is considered flexible enough to adapt to a variety of realistic subsurface structures during the joint inversion process.

In the following example, the spherical semivariogram model is used to describe spatial correlation as a function of lag distance $h$ :

$\gamma(h)=\left\{\begin{array}{cc}c \cdot\left[1.5 \cdot(h / a)-0.5 \cdot(h / a)^{3}\right] & \text { for } h<a \\ c & \text { for } h \geq a\end{array}\right.$

Here, $a$ is the correlation length, and $c$ is the sill value. Two-dimensional orientation and geometric anisotropy are defined through a rotation angle $\beta$ and an anisotropy factor $\omega$ applied to the range parameter $a$, respectively. Sequential Gaussian simulation [Deutsch and Journel, 1992] is used to generate realizations of spatially correlated permeability fields that are consistent with the semivariogram model (7) and are conditioned on permeability values estimated at prelocated pilot points. Note that the number and positions of pilot points should be related to the resolution of the observation data and the degree of heterogeneity expected. Moreover, the choice of pilot points affects the degree of freedom of the inverse problem and averaging occurring during the inversion (i.e., the flexibility with which heterogeneity can be represented, and thus the spatial correlation of the final residuals (for an excellent discussion of these issues, see Moore and Doherty [2006]).

\section{INVERSE METHODOLOGY}

The joint inversion methodology employed here closely follows that presented in Kowalsky et al. [2005], in which the best-estimate parameter set a (holding hydrogeological, petrophysical, and geostatistical parameters) is determined by minimizing the weighted differences between measured (z) and calculated $(F(\mathbf{a}))$ hydrological and geophysical data. Assuming that (1) the final residuals are randomly distributed and can be characterized by a known covariance matrix $\mathbf{C}_{z z}$ (i.e., the contribution of modeling errors to the residuals-which often leads to systematic residuals with significant spatial correlations - is small), (2) measurement errors are uncorrelated (i.e., $\mathbf{C}_{z z}$, is diagonal), and (3) no prior information about the parameters to be estimated is available, the objective function to be minimized has the following form:

$$
O F(\mathbf{a})=[\mathbf{z}-F(\mathbf{a})]^{T} \mathbf{C}_{z z}^{-1}[\mathbf{z}-F(\mathbf{a})]
$$

The forward operator $F($ a) represents three models, namely (1) the hydrological model (Equations 1-5), which is combined with (2) the spherical semivariogram model (Equation 7) used for the generation of random, spatially correlated permeability fields using geostatistical simulation, and (3) the geophysical model for calculating the travel time of GPR waves between transmitting and receiving antennas (Equation 6). The input parameters contained in vector a include hydrological parameters (reference permeability and capillary-strength parameters, permeability modifiers at pilot points), petrophysical parameters (dielectric constant and mixing exponent), and geostatistical parameters (correlation length, orientation angle, and anisotropy ratio); Table 1 summarizes the parameters to be estimated in the following example; the resulting heterogeneous permeability field is visualized in Figure 1a. The set of parameters to be estimated is obviously limited in this illustrative example. While some parameters (such as the residual liquid saturation) are fixed because of insignificant sensitivity, others (such as the spatial distribution of porosity) may be considered unknown or uncertain and should thus be subjected to parameter estimation in field applications.

Measured data are contained in vector $\mathbf{z}$ and include hydrological observations (flow rates, saturations) and geophysical data (GPR arrival times); Table 2 summarizes the observations used in the following example, with the GPR straight-ray pattern visualized in Figure 2. The covariance matrix $\mathbf{C}_{z z}$ contains the variances of the measurement errors on its diagonal. This approach properly weighs data of different type and quality in a joint inversion.

In the following example, the Levenberg-Marquardt algorithm [Levenberg, 1944; Marquardt, 1963] as implemented in iTOUGH2 [Finsterle, 1999] is used to minimize the objective function (8). Since the permeability fields are created using a random process, multiple inversions will be performed. The non-random components of the soil structure can then be identified by averaging the results from the individual realizations. In addition, the impact of smallscale variability on the estimated parameters can be examined. The approach is demonstrated using synthetically generated data, as discussed in the following section. 


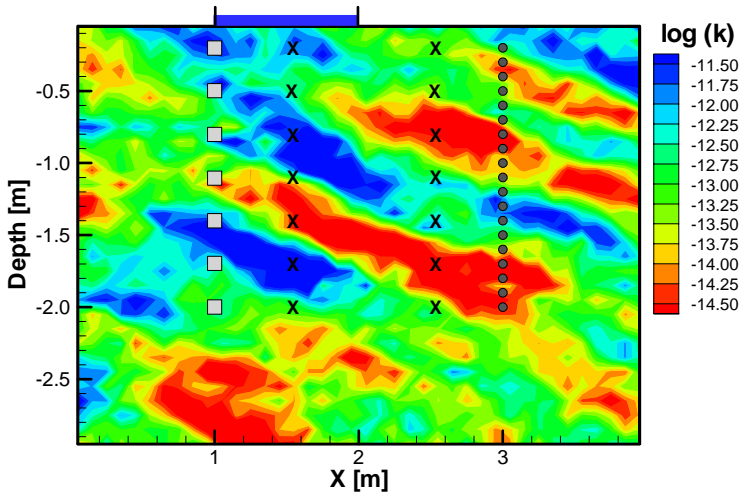

(a)

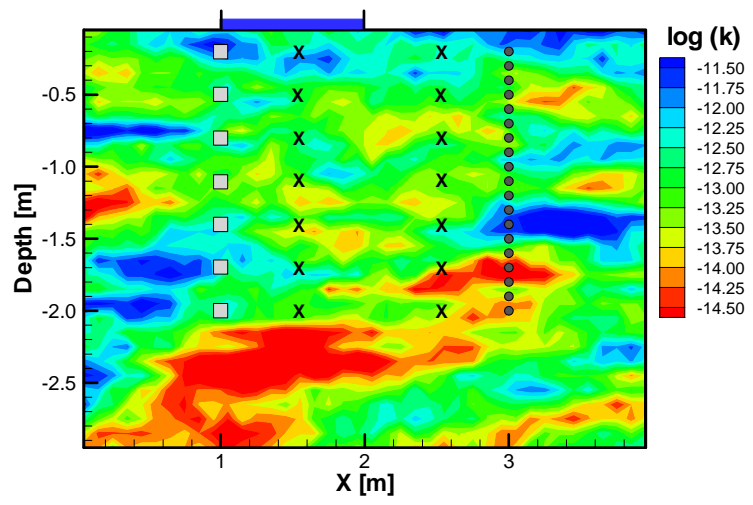

(b)

Figure 1. Spatially structured random permeability field, position of infiltration pond, location of GPR antennas (squares: transmitting; circles: receiving) and position of pilot points (crosses): (a) true field; (b) initial field prior to joint inversion.

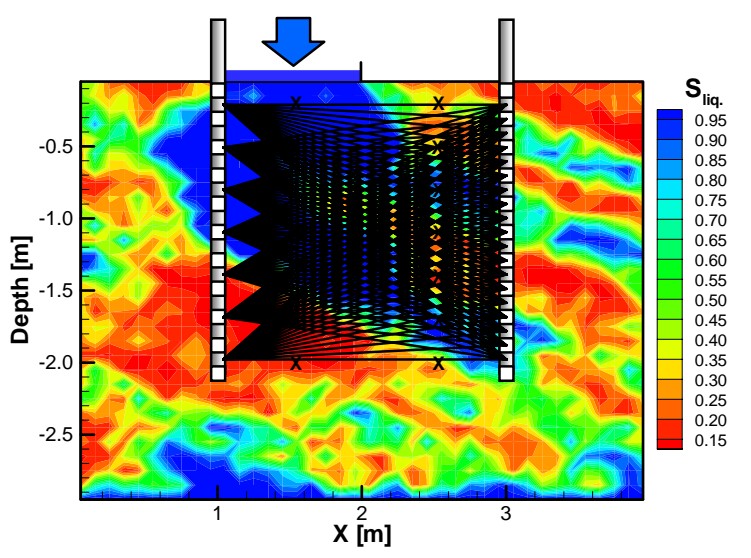

Figure 2. Liquid saturation distribution after one day of water release, locations of neutron probes in boreholes (squares), and GPR straight-ray paths used for inversion.
Table 1. Hydrological, Petrophysical, and Geostatistical Parameters Fixed and Estimated by Joint Inversion of Hydrological and Geophysical Data

\begin{tabular}{|c|c|c|}
\hline Description & $\begin{array}{l}\text { Param. } \\
\text { Value }\end{array}$ & $\begin{array}{c}\text { Fixed or } \\
\text { To Be } \\
\text { Estimated }\end{array}$ \\
\hline \multicolumn{3}{|c|}{ Hydrological parameters, Equations (1)-(5) } \\
\hline $\begin{array}{l}\text { Reference absolute } \\
\text { permeability, } \log \left(k_{r e f}\left[\mathrm{~m}^{2}\right]\right)\end{array}$ & -13.0 & estimated \\
\hline $\begin{array}{l}\text { Permeability modifiers at pilot } \\
\text { points, } \log \left(k / k_{r e f}\right)\end{array}$ & 0.0 & estimated \\
\hline $\begin{array}{l}\text { Reference capillary-strength } \\
\text { parameter, } \log \left(1 / \alpha_{\text {ref }}[\mathrm{Pa}]\right)\end{array}$ & 3.0 & estimated \\
\hline Porosity, $\phi$ & 0.3 & estimated \\
\hline Residual liquid saturation, $S_{l r}$ & 0.08 & fixed \\
\hline van Genuchten parameter, $m$ & 0.63 & fixed \\
\hline \multicolumn{3}{|c|}{ Petrophysical parameters, Equation (6) } \\
\hline Mixture exponent, $n$ & 0.5 & estimated \\
\hline Dielectric constant, solid, $\kappa_{S}$ & 4.0 & estimated \\
\hline Dielectric constant, water, $\kappa_{w}$ & 80.0 & fixed \\
\hline Dielectric constant, air, $\kappa_{a}$ & 1.0 & fixed \\
\hline \multicolumn{3}{|c|}{ Geostatistical parameters, Equation (7) } \\
\hline Correlation length, $a[\mathrm{~m}]$ & 0.4 & estimated \\
\hline Geometric anisotropy ratio, $\omega$ & 5.0 & estimated \\
\hline Orientation, $\beta\left[^{\circ}\right]$ & 70.0 & estimated \\
\hline Sill value, $\log (c)$ & 1.0 & Fixed \\
\hline
\end{tabular}

\section{EXAMPLE}

The joint inversion methodology is demonstrated using a synthetic field experiment. In the simulation, water is released from a pond into a highly heterogeneous but structured vadose zone with a shallow water table at a depth of $3 \mathrm{~m}$. The initial saturation distribution is in capillary-gravity equilibrium with the water table. The infiltration rate is measured as the water level in the pond is maintained at $2 \mathrm{~cm}$ for 3 days. Two monitoring boreholes are equipped with neutron probes for water content measurements; the same boreholes are used for GPR surveys (see Figure 2). Measurements are taken prior to infiltration, and once every day for 5 days. Gaussian noise is added to the synthetic data using the standard deviations given in Table 2.

Permeabilities at the 14 pilot points were initialized to a uniform value of $10^{-14} \mathrm{~m}^{2}$, yielding a heterogeneous permeability field (Figure $1 \mathrm{~b}$ ) that is substantially different from the true field (Figure 1a). Specifically, the area between the two boreholes is essentially homogeneous with no indication of dipping structures (the orientation parameter $\beta$ was set to $90^{\circ}$, i.e., horizontal bedding). The infiltration and redistribution of water in this initial system leads 
to flow rates and saturation patterns (and thus GPR arrival times) that deviate significantly from the measured data.

Table 2. Hydrological and Geophysical Data Used for Joint Inversion

\begin{tabular}{|c|c|c|}
\hline Description & $\begin{array}{l}\text { Number of } \\
\text { Data Points }\end{array}$ & $\begin{array}{l}\text { Data } \\
\text { Error }\end{array}$ \\
\hline \multicolumn{3}{|c|}{ Hydrological data } \\
\hline $\begin{array}{l}\text { Release rate from } \\
\text { infiltration pond }\end{array}$ & 6 daily averages & 0.1 L/day \\
\hline $\begin{array}{l}\text { Neutron probe water } \\
\text { content measurements } \\
\text { in two boreholes }\end{array}$ & $\begin{array}{c}6 \text { daily } \\
\text { measurements at } \\
2 \times 20 \text { Points } \\
\end{array}$ & 0.02 \\
\hline \multicolumn{3}{|c|}{ Geophysical data } \\
\hline GPR travel times & $\begin{array}{c}6 \text { daily surveys } \\
\text { involving } \\
133 \text { antenna pairs }\end{array}$ & $0.5 \mathrm{~ns}$ \\
\hline
\end{tabular}

The Levenberg-Marquardt algorithm as implemented in iTOUGH2 is used to minimize the weighted leastsquares objective function (8). During the iterative inversion procedure, permeability modifier values at the pilot points are adjusted to capture local low- and high-permeability zones. At the same time, the parameters of the geostatistical model (orientation, correlation length, and anisotropy ratio) are updated along with porosity, reference permeability, and capillary strength. Adjusting these parameters affects the infiltration rate as well as the time-dependent saturation values measured by the neutron probe and reflected by the GPR data. Mismatches between the observed and calculated GPR data are further reduced by determining the unknown parameters of the petrophysical models.

An estimated permeability field obtained by the joint inversion process is shown in Figure 3a. The distribution not only exhibits the random nature of subsurface heterogeneity, but also includes the more deterministic, larger-scale features. Most importantly, the result is site-specific in that low- and high-permeability regions are not randomly positioned (as would be the case if standard geostatistical simulation were employed). Because the inversion is conditioned on information contained in the hydrological and geophysical data, these features closely match the actual soil structure (see Figure 1a). Recall that no prior information about the permeability field was available for direct conditioning of the permeability field in the traditional, geostatistical sense.

The resulting permeability field depends on the seed number used by the random number generator implemented in the geostatistical simulation program. Changing the seed number affects the small-scale random structure of the initial permeability field as well as any field created during the optimization process, as the statistical properties are updated. To remove the impact of this unidentifiable component, multiple joint inversions were performed with different seed numbers. The median of the resulting permeability fields is shown in Figure 3b. The salient features of the soil structure are well identified between the two boreholes, whereas the averaging process results in an unstructured effective permeability in the regions where no geophysical data are available. This is also reflected by the standard deviation shown in Figure 3c.

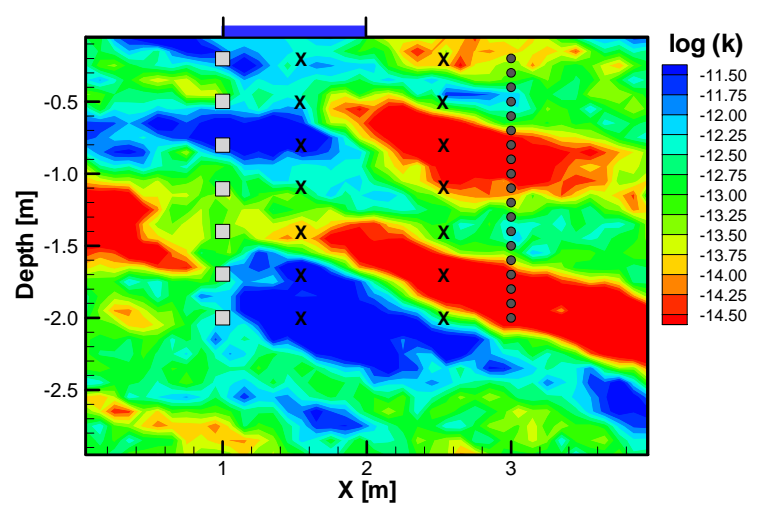

(a)

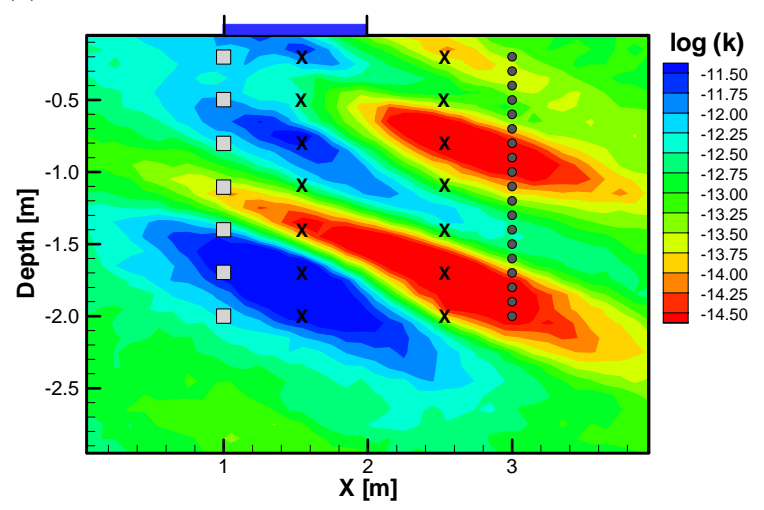

(b)

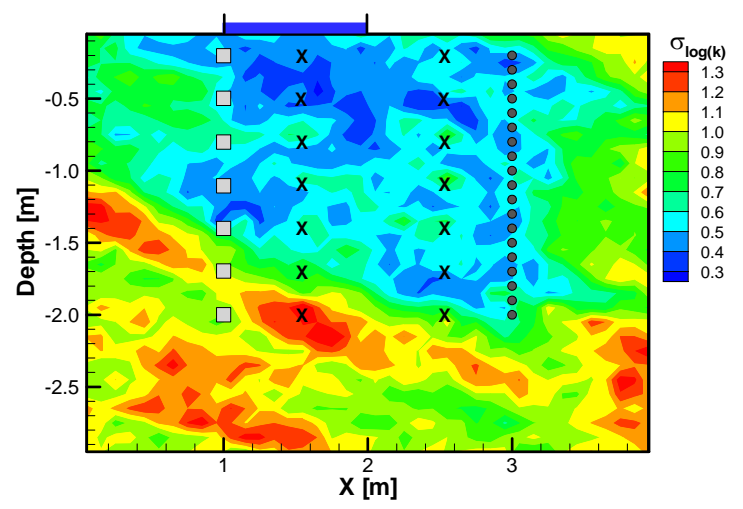

(c)

Figure 3. Permeability field estimated by joint inversion; (a) single realization; (b) median from 20 realizations; (c) standard deviation showing estimation uncertainty. 


\section{CONCLUSIONS}

Subsurface fluid flow and contaminant transport are highly affected by the spatial distribution of unsaturated hydraulic properties as well as the in situ saturation distribution. Characterization of this distribution at a given site is challenging as both random and deterministic subsurface features need to be identified. Standard parameter estimation methods assume that the soil structure is given, i.e., flow and transport properties are determined for predefined zones. Any error in the predefined soil structure is likely to lead to substantial estimation biases in the hydrogeologic parameters.

We developed a joint inversion methodology that takes advantage of the high-resolution information contained in geophysical data, and combines it with process information contained in hydrological data. In addition, the soil structure is assumed to be unknown and parameterized using geostatistical concepts. The inversion process concurrently matches hydrological and geophysical data by jointly estimating hydrogeological, geostatistical, and petrophysical parameters. This approach provides the site-specific soil structure and its properties, capturing both the random and systematic components of the heterogeneity.

Additional numerical experiments and applications to field sites are being performed to examine the strengths and limitations of the proposed approach.

\section{ACKNOWLEDGMENT}

We would like to thank Curt Oldenburg and Chris Doughty for reviewing the manuscript. This work was supported by Laboratory Directed Research and Development (LDRD) funding from Berkeley Lab, provided by the Director, Office of Science, of the U.S. Department of Energy under Contract No. DEAC02-05CH11231.

\section{REFERENCES}

Ansoult, M., L. W. DeBacker, and M. Declrercq (1984), Statistical relationship between apparent dielectric constant and water content in porous media. Soil Sci. Soc. Am. J., 48, 47-50.

Binley, A., G. Cassiani, R. Middleton, and P. Winship (2002), Vadose zone flow model parameterisation using cross-borehole radar and resistivity imaging. Journal of Hydrology, 267(3-4), 147-159.

Carle, S. F., and G. E. Fogg (1996), Transition probability-based geostatistics. Math. Geol., 28(4), 453-476.

Deutsch, C. V., and A. G. Journel. 1992. GSLIBGeostatistical Software Library and User's Guide. Oxford University Press, New York.
Finsterle, S. (1999), iTOUGH2 User's Guide, LBNL40040, Lawrence Berkeley National Laboratory, Berkeley, CA.,http://www-esd.lbl.gov/iTOUGH2.

Finsterle, S. (2005), Multiphase inverse modeling: Review and iTOUGH2 applications. Vadose Zone Journal, 3(3), 747-762.

Gomez-Hernandez, J. J., A. Sahuquillo, and J. E. Capilla (1997), Stochastic simulation of transmissivity fields conditional to both transmissivity and piezometric data-1, Theory. Journal of Hydrology, 203, 162-174.

Huisman, J. A., S. S. Hubbard, J. D. Redman, and A. P. Annan (2003), Measuring soil water content with Ground Penetrating Radar: A review. Vadose Zone Journal, 2, 476-491.

Kowalsky, M. B., S. Finsterle, J. Peterson, S. S. Hubbard, Y. Rubin, E. Majer, A. Ward, and G. Gee (2005), Estimation of field-scale soil hydraulic and dielectric parameters through joint inversion of GPR and Hydrological data. Water Resources Research, 41, W11423, doi:11410.11029/12005WR004237.

Kowalsky, M. B., S. Finsterle, and Y. Rubin (2004), Estimating flow parameter distributions using ground-penetrating radar and hydrological measurements during transient flow in the vadose zone. Advances in Water Resources, 27(6), 583-599.

Lambot, S., M. Antoine, I. van den Bosch, E. C. Slob, and M. Vanclosster (2004), Electromagnetic inversion of GPR signals and subsequent hydrodynamic inversion to estimate effective vadose zone hydraulic properties. Vadose Zone Journal, 3, 1072-1081.

Levenberg, K. (1944), A method for the solution of certain problems in least squares. Q. Appl. Math., 2, 164-168.

Leverett, M. C. (1941), Capillary behavior in porous solids. Trans. Soc. Pet. Eng. AIME, 142, 152-169.

Marquardt, D. (1963), An algorithm for least squares estimation of non-linear parameters. SIAM J. Appl. Math., 11, 431-441.

McLaughlin, D., and L. R. Townley (1996), A reassessment of the groundwater inverse problem. Water Resources Research, 32(5), 1131-1161.

Molz, F. J., H. H. Liu, and J. Szulga (1997), Fractional Brownian motion and fractional Gaussian noise in subsurface hydrology-A review, presentation of fundamental properties, and extensions. Water Resources Research, 33(10), 22732286.

Moore, C., and J. Doherty (2006), The cost of uniqueness in groundwater model calibration. Advances in Water Resources, 29, 605-623.

Neuman, S. P. (1990), Universal scaling of hydraulic conductivity and dispersivity in geologic media. Water Resources Research, 26(8), 1749-1758. 
Pruess, K., B. Faybishenko, and G. S. Bodvarsson (1999a), Alternative concepts and approaches for modeling flow and transport in thick unsaturated zones of fractured rocks. Journal of Contaminant Hydrology, 38(1-3), 281-322.

Pruess, K., C. Oldenburg, and G. Moridis. (1999b), TOUGH2 User's Guide, Version 2.0, Report LBNL-43134, Lawrence Berkeley National Laboratory, Berkeley, Calif.., http://wwwesd.lbl.gov/TOUGH2.

RamaRao, B. S., G. de Marsily, and M. G. Marietta (1995), Pilot point methodology for automated calibration of an ensemble of conditionally simulated transmissivity fields: 1 . Theory and computational experiments. Water Resources Research, 31(3), 475-493.

Richards, L. H. (1931), Capillary conduction of liquids through porous mediums. Physics, 1, 318333.

Roth, K. R., R. Schulin, H. Flühler, and W. Attinger (1990), Calibration of time domain reflectometry for water content measurement using a composite dielectric approach. Water Resources Research, 26(10), 2267-2273.
Rucker, D. F., and T. P. A. Ferré (2004), Parameter estimation for soil hydraulic properties using zero-offset borehole radar: Analytical method. Soil Sci. Soc. Am. J., 68, 1560-1567.

van Genuchten, M. T. (1980), A closed-form equation for predicting the hydraulic condurctivity of unsaturated soils. Soil Sci. Soc. Am. J., 44, 892898.

Yeh, T.-C. J., and J. Šimunek (2002), Stochastic fusion of information for characterizing and monitoring the vadose zone. Vadose Zone Journal, 1, 207-221.

Zimmerman, D. A., G. de Marsily, C. A. Gotway, M. G. Marietta, C. L. Axness, R. L. Beauheim, R. L. Bras, J. Carrera, G. Dagan, P. B. Davies, D. P. Gallegos, A. Galli, J. Gómez-Hernández, P. Grindrod, A. L. Gutjahr, P. K. Kitanidis, A. M. Lavenue, D. McLaughlin, S. P. Neuman, B. S. RamaRao, C. Ravenne, and Y. Rubin (1998), A comparison of seven geostatistically based inverse approaches to estimate transmissivities for modeling advective transport by groundwater flow. Water Resources Research, 34(6), 1373-1413. 